\title{
Gero Erdmann-Preis für vergleichende Area-Forschung 2018
}

\section{Gert Pickel}

Online publiziert: 28. Januar 2019

(C) Springer Fachmedien Wiesbaden GmbH, ein Teil von Springer Nature 2019

Erst einmal danke ich im Namen der Preisstifter der Sektion Vergleichende Politikwissenschaft für die Möglichkeit, diesen Nachwuchsförderungspreis in diesem Rahmen zu überreichen. Da der Zeitplan einer Mitgliederversammlung immer eng bemessen ist, will ich Sie alle hier auch nicht zu lange von der wohlverdienten Pause, vor dem - wie auch immer gearteten - Abendprogramm dieses Tages abhalten.

Vor zwei Jahren haben wir - dies ist der Arbeitskreis Demokratieforschung der DVPW, das German Institute of Global and Area-Studies, kurz GIGA genannt, und die Zeitschrift für Vergleichende Politikwissenschaft - begonnen einen uns geschätzten Kollegen zu ehren - Gero Erdmann. Viel zu früh im Sommer 2014 verstorben, war er einer der Mitbegründer der Zeitschrift für Vergleichende Politikwissenschaft - und vieles mehr. Mir und vielen von uns hier Anwesenden und Nichtanwesenden war er aber vor allem immer ein guter Freund und Kollege. Aus diesem Anlass haben sich der Arbeitskreis Demokratieforschung, dem Gero lange angehörte, das German Institute of Global and Area-Studies in Hamburg, wo Gero lange Jahre in zentralen Funktionen arbeitete und die Zeitschrift für Vergleichende Politikwissenschaft, in der er aktiv als Redakteur beteiligt war, entschlossen, den hier zu übergebenden Förderpreis auszuloben. Wir dachten, es wäre eine gute Idee gerade das wissenschaftliche Andenken an Gero dadurch zu würdigen, dass wir die von ihm so stark geprägte vergleichende Area-Forschung in die Zukunft verlängern.

Der Gero Erdmann-Preis für vergleichende Area-Forschung wird regelmäßig alle zwei Jahre an junge Wissenschaftler vergeben, deren Arbeiten sich durch das $\mathrm{Zu}$ sammenspiel von wissenschaftlicher Exzellenz, einem klaren komparativen Ansatz und eine Berücksichtigung der Area-Forschung zusammensetzen. Der Preis ist mit

Prof. Dr. G. Pickel ( $\square)$

Professur für Religions- und Kirchensoziologie, Universität Leipzig, Theologische Fakultät, Abteilung Religions- und Kirchensoziologie, Martin-Luther-Ring 3, 04109 Leipzig, Deutschland E-Mail: pickel@rz.uni-leipzig.de 
Von links: Gert Pickel, Bartek Pytlas (Foto: DVPW)

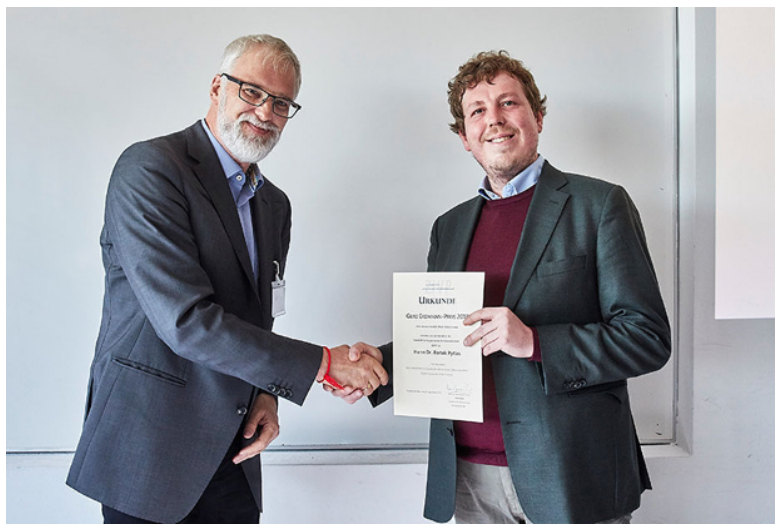

$1500 €$ dotiert. Die diesjährigen Juroren - wir müssen zugeben, leider alle männlich - waren, neben meiner Wenigkeit für die Zeitschrift für Vergleichende Politikwissenschaft, Dr. Toralf Stark für den Arbeitskreis Demokratieforschung und Dr. Thomas Richter für das German Institute of Global and Area-Studies in Hamburg.

Aus den eingereichten Arbeiten, alle ausgesprochen interessant zu lesen, wurde die Dissertationsschrift von Herrn Dr. Bartek Pytlas ausgewählt. Sie beschäftigt sich mit der hochaktuellen Thematik ,Radical Right Parties in Central and Eastern Europe“, Untertitel „Mainstream party competition and electoral fortune“. Betreuer der Dissertation, die an der Europa Universität Viadrina in Frankfurt (Oder) entstanden ist, ist der auf diesem Gebiet ja hocheinschlägige Kollege Michael Minkenberg.

Die vorliegende Untersuchung beschreibt und analysiert unter Bezug auf inhaltlich ausgereifte Fallstudien zu Ungarn und Polen, aber auch über breiter gefasste systematische Vergleiche, Strategien wie Bezugsnarrative rechtsextremer Parteien und begründet deren Erfolg oder Misserfolg in Parteiensystemen. Sie stellt einen Bedeutungsgewinn rechtsextremer Narrative aufgrund eines Bedeutungsgewinns der entsprechenden - schon lange durch rechtsextreme Parteien besetzte - Themen fest. Dieser Bedeutungszuwachs äußert sich im Entstehen oder dem Relevanzgewinn soziokultureller Spannungslinien, deren Narrative über den Parteienwettbewerb Eingang auch in das Repertoire von sogenannten ,Mainstream“-Parteien finden. Damit verlängert sich die Wirkung rechtsextremer Diskurse über deren Parteienkonstellationen hinaus, was gelegentlich für diese zum Problem mit Blick auf den eigenen Erfolg im Parteiensystem wird.

Herr Dr. Pytlas nimmt sich mit seiner Arbeit einerseits einer besonders aktuellen Fragestellung der Politikwissenschaft an, andererseits richtet er seinen Blick auf eine Region, die für längere Zeit etwas aus dem Blick der politikwissenschaftlichen Forschung geraten war - Mittel- und Osteuropa. Durch eine sehr gut gelungene Verbindung von systematischer komparativer Analyse mit Area-Sensibilität gelingt es Herrn Dr. Pytlas in genau der von den Juroren gewünschten Weise die Forderung einer Weiterführung der vergleichenden Area-Forschung zu erfüllen. Speziell das versierte Zusammenspiel von quantitativ-empirischer Arbeit, Hinterfragung von Narrativen und theoretischer Fundierung hat uns überzeugt. Fasst man es zusammen, 
so kann man sagen: Bei der Arbeit von Bartek Pytlas handelt es sich um ein mit vielfältiger Methodenkompetenz gearbeitetes empirisches Werk, welches in exzellenter Manier die Kriterien der Comparative Area-Studies erfüllt und sie weiterführt.

Ich gratuliere Herrn Pytlas im Namen der Juroren, wie des Arbeitskreises Demokratieforschung, der Zeitschrift für Vergleichende Politikwissenschaft und des German Institute of Global and Area-Studies. Als Appetithappen für das lesenswerte Buch, welches in einer einschlägigen politikwissenschaftlichen Schriftenreihe bei Routledge erschienen ist, wird Herr Dr. Pytlas eine Essenz seiner Ergebnisse und Ideen in einem der nächsten Hefte der Zeitschrift für Vergleichende Politikwissenschaft veröffentlichen. Ich kann ihnen die Lektüre des Beitrages, wie auch des Buches nur sehr ans Herz legen.

Ganz herzlich danken möchte ich zum Abschluss noch einmal dem Sprecherrat der Sektion Vergleichende Politikwissenschaft, der diese Verleihung möglich machte. $\mathrm{Zu}$ ihm besteht eine lange - nicht nur persönliche - Verbindung und wir hoffen, dass sich diese auch weiterhin in einer wechselseitigen Win-Win-Situation fortsetzt. Zum Abschluss bleibt es mir nur Sie - neben der Einladung zur Einreichung weiterer guter vergleichender Papiere für die Zeitschrift für Vergleichende Politikwissenschaft - auch zur Einreichung ihrer Dissertationen, oder von Dissertationen, die sie betreut haben, für den nächsten Gero Erdmann-Preis einzuladen. 